\title{
Multiple and contrasting pressures determine intraspecific phytochemical variation in a tropical shrub
}

Andrea Glassmire ( $\sim$ glssmr33@gmail.com )

Louisiana State University https://orcid.org/0000-0003-3286-5642

\section{Walter Carson}

University of Pittsburgh

Angela Smilanich

University of Nevada Reno

Lora Richards

University of Nevada Reno

Christopher Jeffrey

University of Nevada Reno

Craig Dodson

University of Nevada Reno

Casey Philbin

University of Nevada Reno

Garcia Humberto

Organization for Tropical Studies

\section{Lee Dyer}

University of Nevada Reno

\section{Research Article}

Keywords: intraspecific variation, plant chemistry, trophic interactions, resource availability, soil heterogeneity

Posted Date: March 3rd, 2022

DOI: https://doi.org/10.21203/rs.3.rs-1347564/v1

License: (c) (1) This work is licensed under a Creative Commons Attribution 4.0 International License.

Read Full License 
2 Multiple and contrasting pressures determine intraspecific phytochemical variation in a 3 tropical shrub

4 Andrea E. Glassmire ${ }^{1}$, Walter P. Carson ${ }^{2}$, Angela M. Smilanich ${ }^{3}$, Lora A. Richards ${ }^{3,4}$,

5 Christopher S. Jeffrey ${ }^{4,5}$, Craig D. Dodson ${ }^{4,5}$, Casey S. Philbin ${ }^{4,5}$, Garcia L. Humberto ${ }^{6}$, and Lee

6 A. Dyer ${ }^{3,4}$

$7 \quad{ }^{1}$ Department of Biological Sciences, Louisiana State University, Baton Rouge, LA USA

$8 \quad{ }^{2}$ Department of Biological Sciences, University of Pittsburgh, Pittsburgh, PA USA

$9 \quad{ }^{3}$ Department of Biology, University of Nevada, Reno, Reno, NV USA

$10{ }^{4}$ Hitchcock Center for Chemical Ecology, University of Nevada, Reno, Reno, NV USA

$11{ }^{5}$ Department of Chemistry, University of Nevada, Reno, Reno, NV USA

$12{ }^{6}$ Organization for Tropical Studies, La Selva Research Station, Costa Rica

13 *Corresponding Author: Email: glssmr33@gmail.com, Phone: (814) 558-9764

14 Running Head: Intraspecific phytochemical variation

15

16 Author Contribution

17 A.E.G. wrote the first draft of the manuscript. W.P.C., L.A.D., A.M.S., and L.A.R. contributed 18 substantially to revisions. A.E.G., L.A.D., A.M.S, C.S.J., and G.L.H. generated hypotheses and

19 designed experiments. L.A.D., A.M.S., and C.S.J. funded experiments. A.E.G., A.M.S., and

20 G.L.H. collected the field data. A.E.G., C.S.J., C.D.D., and C.S.P. conducted chemical analyses.

21 A.E.G., L.A.D., and L.A.R. conducted statistical analyses. G.L.H. identified Piper imperiale

22 plants and Eois apyraria caterpillars in the field. 
23 Abstract: Intraspecific phytochemical variation across a landscape can cascade up trophic

24 levels, potentially mediating the composition of entire insect communities. Surprisingly, we

25 have little understanding of the processes that regulate and maintain phytochemical variation

26 likely because these processes are complex and operate simultaneously both temporally and

27 spatially. To assess how phytochemistry varies within species, we tested the degree to which

28 resource availability, contrasting soil type, and herbivory generate intraspecific chemical

29 variation in growth and defense of the tropical shrub, Piper imperiale (Piperaceae). We

30 quantified changes in both growth (e.g., nutritional protein, above- and below-ground biomass)

31 and defense (e.g., imide chemicals) of individual plants using a well-replicated fully factorial

32 shade-house experiment in Costa Rica. We found that plants grown in low light and nutrient-

33 poor residual soil had reduced foliar protein, while those grown in high light and richer old

34 alluvial soil had increased protein. Thus, investment into growth was determined by resource

35 availability and soil composition. Surprisingly, we found that chemical defenses decreased in

36 response to herbivory. We also found that changes in plant protein were more plastic compared

37 to plant defense, indicating that constitutive defenses may be relatively fixed, and thus an

38 adaptation to chronic herbivory that is common in tropical forests. We demonstrate that

39 intraspecific phytochemical variation of $P$. imperiale is shaped by resource availability from

40 light and soil type. Because environmental heterogeneity occurs over small spatial scales (tens of

41 meters), herbivores may be faced with a complex phytochemical landscape that may regulate

42 how much damage any individual plant sustains.

44 Keywords: intraspecific variation, plant chemistry, trophic interactions, resource availability,

45 soil heterogeneity 
Introduction

47 Phenotypic plasticity determines the degree of intraspecific variation in key traits, which in

48 turn modifies species interactions across food webs (Turcotte and Levine 2016). Parasites can

49 cause changes in the chemical profiles of their hosts, and this occurs in a broad array of

50 phylogenetically distinct taxa and in nearly all ecosystems. For example, herbivory, changes in

51 resource availability, or both can induce chemical defenses in algae (Van Alstyne 1988, Cronin

52 and Hay 1996), freshwater macrophytes (Morrison and Hay 2011), endophytes (Saikkonen et al.

53 2013), and herbs, shrubs, and trees (Fowler and Lawton 1985, Kessler and Kalske 2018, Moore

54 et al. 2014, Glassmire et al. 2016, Dyer et al. 2018). Most studies on intraspecific variation in

55 phytochemical defenses have either focused on specific mechanisms in isolation, such as induced

56 defenses, or have only examined interspecific differences among species (Feeny 1976, Rhoades

57 and Cates 1976, Herms and Matson 1992, Hay 2002, Fink 2007, Smilanich et al. 2016). This

58 metabolic variation that occurs within species is a key dimension of diversity, but surprisingly,

59 the putative ecological processes that determine intraspecific variation in plant defense are rarely

60 compared experimentally and are thus poorly understood (Morrison and Hay 2011, Hahn and

61 Maron 2016, Hunter 2016). Phytochemical defenses are hypothesized to shape herbivore host

62 plant use and may underlie patterns of diversification in phytophagous insects (Endara et al.

63 2017). Across most taxa and ecosystems, the processes that cause intraspecific variation in

64 phytochemical landscapes are mostly unresolved, even for abundant species (Hunter \& Price

65 1992, Hunter 2016). Here, we directly compare different sources of intraspecific variation in

66 chemical defense by experimentally quantifying the degree to which key limiting resources (light 
67 and nutrients), enemies, and soil type, mediate intraspecific variation in nutritional and defensive 68 secondary metabolites.

69 Intraspecific phytochemical variation is shaped by top-down and bottom-up pressures,

70 and it is likely that simultaneous effects of resource availability and herbivory impact plant

71 fitness across the landscape (Hunter \& Price 1992, Massad et al. 2012, Hahn and Maron 2016,

72 Hunter 2016). Variation in resource availability acts as a bottom-up pressure that regulates

73 growth and defense (Coley et al. 1985, Loomis 1932, Herms and Mattson 1992, Stamp 2004).

74 Similarly, changes in availability of both light and nutrients are hypothesized to cause tradeoffs

75 between plant growth and defense. For example, when plants obtain nutrients and light beyond

76 their physiological demands, then resources can be diverted from growth toward defense. In

77 contrast, herbivory is a top-down pressure that can alter plant metabolism; the focus of these

78 effects has been on changes in plant defensive compounds and relies on the assumptions that

79 certain compounds are metabolically expensive and that they are produced to mitigate additional

80 damage from parasites (Myers 1988, Agrawal and Karban 1999, Dicke and Hilker 2003). While

81 we have clear predictions of individual responses to variation in both resource availability and

82 herbivory, we do not have clear predictions of how these top- and bottom-up processes interact.

83 Thus, we do not have a realistic assessment of what underlies intraspecific phytochemical

84 variation. Even more, some of these factors may have contrasting effects that likely negate the

85 effects of any single process or factor. Overall, there is a compelling need to evaluate the

86 individual and interactive effects of abiotic and biotic processes on intraspecific phytochemical

87 variation.

88 A second key gap in resolving the putative ecological processes that determine intraspecific

89 variation in plant defense is understanding the role of soil heterogeneity on phytochemical 
90 variation (Fine et al. 2006, Cacho and Strauss 2014, Glassmire et al. 2017). Soils are dynamic

91 and heterogeneous across the landscape because of changes in chemical and physical properties

92 due to climate, parent material, topography, the resident plant and microbial community, and

93 many other factors (Clark et al. 1998, Laliberte et al. 2013, van der Putten et al. 2013).

94 Interactions between plants and their soil microbes create positive and negative soil feedbacks

95 (e.g., Bever 2003, Pendergast et al. 2013) to such an extent that they can alter growth, leaf

96 chemistry, and allelopathic potential among co-occurring plant species (Mieners et al. 2017).

97 Contrasting soil type likely underlies a considerable proportion of intraspecific variation in plant

98 defensive chemistry and growth, although it is rarely considered in studies of intraspecific

99 phytochemical variation (Massad et al. 2012, Hunter 2016). Resolving this gap requires

100 conducting controlled experiments that manipulates soil type, in addition to resource availability

101 and herbivory to obtain a more rigorous understanding of how ecological processes mediate

102 intraspecific phytochemical variation.

103 We tested the degree to which resource availability, contrasting soil type, and herbivory

104 generate intraspecific chemical variation by manipulating these putative sources of intraspecific

105 phytochemical variation for a common understory shrub, Piper imperiale, (Piperaceae) in a

106 lowland tropical rainforest in Costa Rica. We examined whether there were tradeoffs between

107 plant growth and defense using a full factorial design where we manipulated soil nutrients, light

108 availability, soil type, and the presence or absence of herbivory. We addressed the following

109 questions: (1) What are the relative effects of soil nutrients, light availability, soil type, and

110 herbivory on plant defensive chemistry? (2) To what degree do these factors mediate plant

111 growth? (3) To what degree do these factors interact and contribute to intraspecific variation in

112 growth and defense? Currently, there is a considerable knowledge gap about tradeoffs between 
113 growth and defense among conspecifics and whether these tradeoffs occur when there is

114 variation in resource availability herbivory, and the soil substrate. Each of these processes vary

115 across small spatial scales beneath forest understories and can change phytochemical variation

116 on the scale of meters thus affecting entire communities. Overall, the degree that each of these

117 process shapes intraspecific phytochemical variation is poorly understood.

118 Materials and Methods

119 Plant Study System - We conducted this study in Costa Rica from June - December

1202014 at the La Selva Biological Station $\left(10.4306^{\circ} \mathrm{N}, 84.0070^{\circ} \mathrm{W}\right)$, a lowland rainforest that

121 receives $\sim 4000 \mathrm{~mm}$ of precipitation annually (Clark 2013). Piper imperiale C.DC. (Piperaceae)

122 is an abundant and shade tolerant shrub that is one of 54 co-occurring Piper species at La Selva

123 and is a model plant system to test hypotheses based on chemical defense (Dyer and Palmer

124 2004; Gentry 1990). Its chemical defenses are well described and include two biosynthetically

125 related nitrogen containing imides, sintenpyridone and piplaroxide, (where sintenpyridone is the 126 precursor of piplaroxide, Figure 1), and at least five different sesquiterpenes, all of which likely

127 contribute synergistically to plant defense (Fincher et al. 2008). Piper imperiale invests in

128 relatively lower concentrations of more highly toxic defensive compounds compared to other

129 Piper species (1.7\% dry weight, Fincher et al. 2008, Richards et al. 2010).

130 Experimental Design - We manipulated soil type, nutrient additions, and herbivory using

131 a factorial design that was nested within low and high light levels. Our treatments included: 1)

132 nutrient-poor old alluvial versus a richer residual soil type, 2) low versus high nutrient additions,

133 and 3) presence or absence of herbivores, totaling 16 treatment combinations. Each treatment

134 combination was replicated 10 times for a total of 160 plant individuals that were randomly 
135 assigned to each treatment combination (Figure S1). Individual vegetative cuttings of $P$.

136 imperiale were collected throughout La Selva from 12 different plant populations.

137 Soil type, nutrient additions, and herbivore manipulations - Both light and soil nutrient

138 availability are limiting and highly patchy within understories of closed canopy tropical forests

139 (for light Montgomery and Chazdon 2002, for nutrients see Wright et al. 2011, Pasquini et al.

140 2015). Light availability also influences plant phytochemistry; higher light levels are associated

141 with $17 \%$ increase in total imide content in Piper cenocladum shrubs (e.g. sintenpyridone, $4^{\prime}-$

142 desmethylpiplartine, and cenocladimide, Dyer et al. 2004). We used reflective shade cloth

143 (AgFabric, Corona, CA) to create two levels of light availability (within an 8 X 3 X $3 \mathrm{~m}$

144 enclosure), an ambient level that commonly occur in forest understories, and a deep shade

145 treatment (see below). We measured photosynthetically active radiation throughout the day

146 beneath our two light treatments using H21-002 HOBO micro station data logger with two S-

147 LIA-M003 sensors (MicroDAQ, Ltd., Contoocook, NH, USA). Our low light treatment reduced

148 light to $2 \%$ full sun and ranged from a minimum of $1.2 \mu \mathrm{mol} \mathrm{m}^{-2} \mathrm{~s}^{-1}$ to a maximum of 106.2

$149 \mu \mathrm{mol} \mathrm{m} \mathrm{m}^{-2} \mathrm{~s}^{-1}$. Our high light treatment reduced light to $30 \%$ full sun and ranged from a minimum

150 of $1.2 \mu \mathrm{mol} \mathrm{m}^{-2} \mathrm{~s}^{-1}$ to a maximum of $363.7 \mu \mathrm{mol} \mathrm{m}^{-2} \mathrm{~s}^{-1}$ (t-test, $P<0.001$ ). The low light

151 treatment mimicked deeply shaded understory patches that occur beneath the shrub and herb

152 layer, and a high light treatment that occurs in the understory above this layer (Montgomery and

153 Chazdon 2002). All individual plants were rotated within the light treatment block every two

154 weeks throughout the duration of the experiment to ensure homogeneity in light and

155 environmental conditions of the shade-house. We watered plants daily during the first two weeks

156 of establishment and then twice weekly for weeks three and four. Thereafter, plants received

157 ample ambient precipitation via perforations in the shade cloth. 
We manipulated soil type by collecting the two main soils (alluvial and residual) that 159 occur throughout La Selva (Sancho and Mata 1987). Old alluvial soils are clay soils with

160 intermediate nutrient content, high water holding capacity, and low drainage, whereas residual

161 soils are derived from highly weathered lava flows and are nutrient poor with high drainage

162 (Sancho \& Mata 1987, Clark et al. 1998). Contrasting plant communities occur on these two soil

163 types (for extensive details see Sancho \& Mata 1987, Clark et al. 1998). We collected soil from

164 four different areas for each soil type by excavating soil down to $15 \mathrm{~cm}$. We bulked and

165 thoroughly mixed soil within a soil type before use. Plant individuals from each population were

166 randomly assigned to one of the two soil types and grown for one month in plastic pots $(3.79 \mathrm{~L})$

167 prior to applying the nutrient and herbivory treatments.

168 We manipulated nutrient resources by adding both macro and micronutrients to the soil.

169 Recent research demonstrates that soil nutrients limit plant performance even in deeply shaded

170 forest understories (reviewed by Wright et al. 2011, Griffin et al. 2017). Higher nutrient

171 additions are associated with 3\% increase in total imide content in Piper cenocladum shrubs (e.g.

172 sintenpyridone, 4'- desmethylpiplartine, and cenocladimide, Dyer et al. 2004). We had high (10

173 grams of macro- and micronutrients per $\mathrm{m}^{2}$ ) and low (2 grams of macro- and micronutrients per

$174 \mathrm{~m}^{2}$ ) nutrient addition treatments; macronutrients included NPK 12-8-16 and micronutrients

175 included magnesium, calcium, copper, sulfur, iron, boron, and zinc (Blaukorn classic, Kyiv,

176 Ukraine). Nitrogen was added in two forms, nitrate and ammonium.

177 Finally, we manipulated herbivory using the specialist native herbivore, Eois apyraria, 178 which commonly causes extensive damage on $P$. imperiale (Fincher et al. 2008, Hansen et al. 179 2017). Eois apyraria is a leaf chewer and bioassays have shown conclusively that the defensive 180 sintenpyridone and piplaroxide chemicals reduce the survival and performance of E. apyraria 
181 (Fincher et al. 2008, Richards et al. 2010, Hansen et al. 2017). We placed two caterpillars on

182 plants that were assigned herbivory. We added new caterpillars to plants assigned herbivory

183 whenever old ones pupated, died, or disappeared for the 6-month duration of the experiment. We

184 quantified the absolute amount of herbivore damage per plant, which ranged from 0 - $30 \%$ leaf

185 area reflecting natural levels of damage on P. imperiale (see Fincher et al. 2008).

186 Harvest - Plants were harvested in December 2014, six months after commencing the

187 experiment. Leaf Surface Area (LSA) of freshly harvested leaves was measured using LI-3100

188 Leaf Area (LI-COR, Inc., Lincoln, NE, USA). Total herbivore damage was assessed for all

189 leaves on each plant by quantifying the Leaf Area Removed (LAR) due to herbivory using LI-

1903100 Leaf Area (LI-COR, Inc., Lincoln, NE, USA). Plant stems, roots, and leaves were

191 separated, dried at $28^{\circ} \mathrm{C}$ for three days, weighed, and stored in paper bags at $-28{ }^{\circ} \mathrm{C}$ until

192 chemical analyses were conducted.

$193 \quad$ Foliar defense chemical and protein analyses - Samples of dried leaves from individuals

194 from each of the sixteen shade-house treatments were extracted for quantification using seven-

195 point calibrations. Defensive imide chemical response units are reported as percent dry weight.

196 We modified a chemical extraction protocol specific to Piper imperiale (Dodson et al. 2000).

197 Briefly, $1.00 \mathrm{~g}$ samples aliquot from each plant were extracted with $5 \mathrm{~mL}$ of 1:1 aqueous

198 methanol and $5 \mathrm{~mL}$ of chloroform. This mixture was sonicated for 20 minutes and centrifuged to

199 allow layers to separate. The organic layer was retained before repeating this liquid-liquid

200 extraction two more times by adding $5 \mathrm{~mL}$ of chloroform to the aqueous layer. Organic extracts

201 were pooled and $1.00 \mathrm{~mL}$ aliquots of samples for each plant were combined with commercially

202 available piperine (MilliporeSigma; St. Louis) as an internal standard (final concentration: 80.0

203 ug/L) and analyzed using a Gas Chromatography-Flame Ionization Detector or GC-FID (Agilent 
204 7890A; Santa Clara, CA). Seven-point calibrations (11.72, 23.44, 46.88, 93.75, 187.5, 375.0, and

$205750.0 \mathrm{ug} / \mathrm{L})$ were prepared using imides $1\left(\mathrm{R}^{2}=0.999\right)$ and $\mathbf{2}\left(\mathrm{R}^{2}=0.999\right)$. External standards for

206 analysis were provided by the Joe Richards lab (Colorado Mesa University, Grand Junction, CO;

207 Dyer et al. 2004). The sintenpyridone imides are unstable in solution, so all reported

208 concentrations are estimates (Dodson et al. 2000). For a detailed description of GC methods see

209 Dodson et al. (2000) and Dyer et al. (2001).

210 Total foliar protein concentration for each plant was quantified using the Pierce BCA

211 Protein Assay protocol (Thermo Fisher Scientific, Waltham, MA, USA). Briefly, two mg of

212 dried leaf material was extracted from each plant with $500 \mu \mathrm{L}$ of buffer $(100 \mathrm{mM}$ Tris $\mathrm{pH}$ 7.5,

$213150.00 \mathrm{mM} \mathrm{NaCl}, 10.00 \mathrm{mM} \mathrm{MgCl}{ }^{2}$, and $1 \%$ of SDS). The extracted protein was quantified

214 using a six-point calibration curve of albumin $(0.0,4.0,10.0,20.0,40.0$ and $60.0 \mu \mathrm{g} / \mathrm{mL})$. Foliar

215 protein extractions were replicated for each plant three times to ensure accuracy. The samples

216 and protein standard were analyzed using a SpectraMax M Series Multi-Mode Microplate

217 Reader (Molecular Devices, LLC., Sunnyvale, CA, USA) measuring absorbance at $560 \mathrm{~nm}$. The

218 units of total protein concentration for each sample are reported as the protein mass fraction

219 relative to plant dry weight.

220 Statistical Analyses - All analyses were conducted in program R version 3.6.2 (R Core

221 Team 2018). Analyses were run separately for individuals exposed to high versus low light

222 conditions, due to the availability of one shade-house per light level. We conducted 3-way

223 ANOVAs for each response variable. All analyses included interactions between predictor

224 variables. For response variables that were significant and intercorrelated (i.e. as in biomass or

225 chemical compounds), multivariate analysis of variance (MANOVA) was used to examine

226 responses of plant chemical traits to soil type, nutrient additions, and presence or absence of 
227 herbivores. The response variables for plant biomass (g) were above-ground based on total mass

228 of leaves and stems and below ground based on total mass of roots. The response variables for

229 plant chemistry were protein and the known defense compounds sintenpyridone (1), and

230 piplaroxide (2) calculated as percent dry weight. Profile analysis followed all MANOVAs, which

231 compares effects of predictors for each response variable (Tabachnick \& Fidel 1989; Tabachnick

232 et al. 2001). Residuals of all models were approximately normally distributed. We also report

233 standardized Cohen's $d$ and raw effect sizes to help interpret biological effects of manipulations

234 (Tables S1-S5, Cohen 1994; Torchiano2020). We used the 'effsize' package to calculate Cohen's

$235 \mathrm{~d}$ (Torchiano 2020).

236 We paired traditional MANOVA analyses with structural equation models (SEM using

237 the 'lavaan' and 'blavaan' packages, Rosseel 2012) and hierarchical Bayesian models (using

238 'rjags' package, Plummer 2021) to test specific a priori hypotheses, to examine specific direct

239 and indirect effects, and to estimate relationships among endogenous variables. The hierarchical

240 Bayesian models treated the two light experiments as the upper level of the hierarchy, with all

241 treatment level combinations nested in the lower level; these analyses were used to support

242 inferences about the different results found in the two different light levels. The Bayesian

243 approaches were also utilized to minimize Type II error - providing probabilities of nulls for

244 parameter estimates that were close to zero and that had high alpha values in the MANOVAs or

245 SEMs.

246 Results

247 The results for high versus low light levels are described separately for ease of interpretation and

248 because soil type, nutrients, and herbivore treatment combinations were nested within each light

249 treatment. However, complex differences in the high and low light experiments were clearly 
250 supported by the hierarchical Bayesian models, $95 \%$ credibility intervals did not cross zero

251 (Figure S2), with substantial overall decreases in biomass, protein, and chemical defense for

252 plants in low light versus highlight experiments (Figure S1).Inferences of "nonsignificant"

253 effects were supported by the Bayesian models, with $50 \%$ credibility intervals of parameter

254 estimates for nonsignificant effects crossing zero (Figures S3-S6). For all statistical models, plant

255 biomass and protein were a proxy for growth, while the defensive imide compounds were a

256 proxy for defense.

\section{High Light Conditions}

258 Plant biomass - Nutrient enrichment (low versus high) significantly increased above-

259 ground biomass by $41 \%$ (low nutrients $\bar{x}=16 \mathrm{~g} \pm 1.14 \mathrm{SE}$ compared to high nutrients $\bar{x}=22.5$

$260 \mathrm{~g} \pm 1.54 \mathrm{SE}$; Figure S7) and below-ground biomass by $33 \%$ ( low nutrients $\bar{x}=5.4 \mathrm{~g} \pm 0.38 \mathrm{SE}$

261 compared to high nutrients $\bar{x}=7.1 \mathrm{~g} \pm 0.61 \mathrm{SE}$; Figure 2, Figure S7). Our results demonstrate

262 that soil nutrients increase plant biomass additively (above biomass MANOVA: $F_{1,71}=10.89, P<$

263 0.01; below biomass MANOVA: $F_{1,71}=5.65, P=0.02$; Figure 2, Table S1).

$264 \quad$ Foliar protein and defensive chemistry- A three-way interaction among herbivory, soil

265 type, and nutrient enrichment increased the amount of foliar protein (significant interaction

266 MANOVA: $F_{1,52}=3.31, P=0.07$; Figure 3, Table S4). High levels of herbivory on plants grown in

267 alluvial soils with high nutrient enrichment caused a 55\% increase in foliar protein, however, if

268 these plants were also grown at low nutrient levels, then there was only a $2 \%$ increase in foliar

269 protein (Figure 3, Figure S8). The path analysis confirmed the finding that the damage caused

270 by herbivores increased foliar protein by $13 \%$ (path analysis: $\mathrm{spc}=0.13$; Figure 4 ). Furthermore,

271 because the effect sizes were relatively large across the combination of herbivory, soils, and 
272 nutrients, it is clear that a combination of these factors together caused increases in foliar protein

273 (refer to tables for effect sizes and summary stats, Table S3, Figs. S8-S10).

$274 \quad$ For defensive imide compounds, the path analysis highlighted the strong, positive effect

275 of sintenpyridone on piplaroxide, which is the biosynthetic precursor to the formation of

276 piplaroxide, and thus regulates how much piplaroxide is produced (path analysis: $\mathrm{spc}=0.65$;

277 Figure 4). We also found that nutrient enrichment caused contrasting effects on the levels of 278 imide compounds in residual soils. Specifically, plants grown in residual soil, suffering high

279 levels of herbivory, and low levels of nutrient additions caused reductions in piplaroxide by $99 \%$

280 and sintenpyridone by $58 \%$, whereas high nutrient additions caused an increase in piplaroxide by

$28167 \%$ (significant interaction for piplaroxide MANOVA: $F_{1,52}=3.21, P=0.07$; Figure 5). Plants

282 grown in old alluvial soil, having high levels of herbivory, and low levels of nutrient additions

283 caused reductions in piplaroxide by $33 \%$ and sintenpyridone by $2 \%$, whereas high nutrient

284 additions further reduced piplaroxide by 75\% (significant interaction for piplaroxide MANVOA:

$285 F_{1,52}=3.21, P=0.07$; Figure 5). Counter to our predictions, herbivory alone caused a decline in

286 sintenpyridone by $21 \%$ (path analysis: $\mathrm{spc}=-0.21$; Figure 4, Figures S11-S13). Overall, the

287 production of the defensive piplaroxide compound was mediated by an interaction between soil

288 type, herbivory, and nutrient enrichment and these factors typically vary over fairly small spatial

289 scale throughout forest understories (refer to tables for effect sizes and summary stats, Table S3,

290 Table S5).

\section{Low Light Conditions}

292

Plant biomass - Nutrient enrichment (low versus high) caused above-ground biomass to

293 increase by $31 \%$ (low $\bar{x}=14.7 \pm 1.05 \mathrm{~g}$ vs. high $\bar{x}=19.3 \pm 1.35 \mathrm{~g})$, even under the low light

294 conditions that are characteristic of deeply shaded understories (Tables S1-S2, Figure S7). 
295 Nutrient enrichment did not significantly change below-ground biomass (low $\bar{x}=3.3 \pm 0.25 \mathrm{~g}$

296 vs. high $\bar{x}=4.1 \pm 0.46$ g; Tables S1-S2, Figures S7). Plants established in old alluvial soils

297 having high levels of nutrient enrichment and herbivory caused above-ground plant biomass to

298 decrease by $91 \%$, whereas low levels of nutrient enrichment increased above-ground biomass by

$29996 \%$ (significant interaction MANOVA: $F_{1,71}=3.75, P=0.05$; Figure 2, Tables S1-S2).

$300 \quad$ Foliar protein and defensive chemistry under low light - High nutrient additions caused a

301 reduction of foliar protein by $20 \%$ compared to low nutrient additions (additive effect MANOVA:

$302 F_{1,59}=2.94, P=0.09$; Figure S8, Table S4). Plants grown in residual soils with high levels of

303 herbivory and nutrient additions reduced protein by $167 \%$, although this was not significant

304 (three-way interaction MANOVA: $F_{1,59}=0.13, P>0.7$; Figure 3, Table S4). For chemical

305 defense, sintenpyridone significantly increased piplaroxide, although the strength of this effect

306 decreased under low light compared to high light (path analysis: $\mathrm{spc}=0.46$; Figure 4).

307 Herbivory decreased the amount of sintenpyridone (path analysis: $\mathrm{spc}=-0.18$; Figure 4; Figures

308 S11-S13). There were no significant interactions among nutrients, herbivory, and soil type with

309 regard to leaf protein and the defensive compounds (MANOVA summary statistics in Table S3

310 and Table S5).

311 Discussion

312 The cause of intraspecific phytochemical variation is poorly understood even though we

313 know that the consequences of intraspecific phytochemical variation can cascade up to higher

314 trophic levels. We examined how ecological processes due to resource availability, herbivory,

315 and contrasting soil type influenced patterns of resource allocation between growth or defense

316 for a common and geographically widespread understory shrub, Piper imperiale. We found that

317 high light availability increased plant allocation toward protein production and high levels of 
318 nutrient additions increased plant allocation toward biomass, which is an important indicator of

319 growth. Pervasive interactions among nutrient availability, herbivory, and contrasting soils

320 determined the degree to which plants allocated resources to chemical defense. Surprisingly,

321 herbivory decreased sintenpyridone by $31 \%$ and piplaroxide by $36 \%$. Moreover, we found

322 piplaroxide decreased as much as $99 \%$ in response to herbivory, but only for plants grown in

323 residual soils, and under low nutrient additions. Conversely, high levels of nutrient additions on

324 plants grown in residual soils under high light conditions caused piplaroxide to increase by $67 \%$,

325 but plants grown in old alluvial soils under the same conditions caused piplaroxide to decrease

326 by $75 \%$. These results provide compelling evidence that the effect of herbivory on growth and

327 defense is mediated by soil type and nutrient availability. We also found the imide

328 phytochemicals followed the classic metabolic patterns of constitutive defenses and did not

329 increase in response to herbivory. These results demonstrate unequivocally that the degree to

330 which herbivory changes plant defensive chemistry is determined by variation in resource

331 availability (light and soil nutrients) and soil type. An important consequence of these results is

332 that small-scale variation in key factors such as light availability will likely create mosaics of

333 phytochemical variation among conspecifics. Herbivores must then "sample" this chemical

334 variation when searching for and "evaluating" the suitability of host plants; such variation

335 hinders herbivore acclimation to chemistry and may well lower overall herbivore loads on a plant

336 species across its range (Adler and Karban 1994, Underwood 2014, Barbosa et al. 2009, Hunter

337 2016, Glassmire et al. 2019). In sum, these results demonstrate that to evaluate our hypotheses

338 and understand intraspecific variation in phytochemistry we must simultaneously consider the

339 availability of key limiting resources, soil mosaics, as well as the presence and absence of

340 herbivores. While this adds complexity to our understanding of phytochemistry and plant 
341 defense, it means we cannot ignore abiotic factors that are well known to structure forest

342 understories (Clark et al. 1998, Wright et al. 2011). It also means that herbivores are almost

343 certainly encountering a highly variable phytochemical landscape on relatively small spatial

344 scales that are relevant to these herbivores finding suitable hosts.

346 Light initiated a reduction in plant growth

347 Decreasing light availability caused a reduction in plant biomass and foliar protein, an indicator

348 for plant growth. This, however, was not observed for the defensive compounds. Both the

349 quantity and quality of light availability changes substantially throughout tropical forest

350 understories (e.g., Chazdon \& Fetcher 1984), and can cause major changes in intraspecific

351 chemical variation in Ecuadorian Piper shrubs across small spatial scales (Glassmire et al. 2019).

352 While it is well known that light is a critical limiting resource for understory plants, particularly

353 in tropical forests, oddly, it is rarely measured in studies focused on tradeoffs between growth

354 and defense among conspecifics or heterospecifics (Hahn and Maron 2016, Hahn et al. 2019).

355 Effect sizes for plant growth documented here were substantive; high light levels increased

356 proteins related to plant growth by 55\% (Figure 3, Table S3), whereas low light levels decreased

357 the production of proteins by $-167 \%$ (Figure 3, Table S3). Leaves are costly for plants to produce

358 under low light conditions (Bryant et al. 1983, Lerdau \& Coley 2008), and we found that plants

359 invested in foliar protein in high light environments but not in low light environments.

361 Complex relationships determined the production of defensive compounds

362 The concentrations of secondary metabolites depended substantially on conditions and

363 factors known to vary widely across tropical forest landscapes, specifically resource availability, 
364 soil type, and herbivory. It is worth noting the substantial effect sizes of manipulations on

365 chemistry - the percent dry weight of piplaroxide increased by $67 \%$ when favorable light and soil

366 resources were applied, while this defensive compound decreased by $75 \%$ when plants were

367 exposed to high levels of herbivory (Figure 5). Growing plants in different soil types, exposing

368 them to different levels of herbivore damage, and changing resource availability interacted to

369 modify the production of key defensive compounds. There is little doubt that variation in these

370 conditions and processes will create a very complex chemical landscape in tropical forests,

371 whereby herbivores will encounter host plants that vary substantially in host plant quality. We

372 speculate that this variation is likely the norm for most plant species and future research should

373 explore, not only this possibility, but the consequences of this variation for herbivore

374 assemblages and community processes, such as trophic cascades. What we do know is that even

375 subtle changes ( $0.2 \%$ dry weight) in Piper imide levels can seriously disrupt the immune

376 response of specialist herbivores (Hansen et al. 2017). Moreover, Richards et al. (2010)

377 demonstrated that synergistic effects of three Piper imperiale defensive compounds, including

378 piplaroxide, even at very low amounts $(<0.1 \%$ dry weight $)$, can decrease survival in herbivores

379 by as much as $25 \%$. Prior to this research, it would be difficult to make the case that soil type

380 would be the template upon which there were numerous interactions with herbivory and resource

381 availability, which together determined chemical changes in P. imperiale. These findings show

382 that soil type can be key to determining community interactions (Clark et al. 1998, Fine et al.

383 2006, Cacho and Strauss 2014) and must be considered in future studies (Hahn et al. 2016). The

384 striking differences in forest communities found at La Selva between our two soil types (Clark et

385 al. 1998) may be at least partly due to their impacts on plant chemical defenses, which may

386 cascade up and down trophic webs to mediate forest plant species composition and diversity. 
When there are sharp differences among soil types, selection can lead to the evolution of

388 distinct plant traits adapted to different soils, even when plant species are in fairly close

389 proximity (Fine et al. 2004). For example, Misiewicz and Fine (2014) found evidence of local

390 adaptation to soils, with significant morphological variation, higher levels of genetic

391 differentiation, and lower migration rates among populations of the tropical tree Protium

392 subserratum growing parapatrically on white-sand, brown-sand, and clay soils in Peruvian

393 Amazonia. Similarly, Cacho and Strauss (2014) found the shift for plants to serpentine soils from

394 bare, sparsely vegetated ground was extremely important for plant diversification in the

395 Streptanthoid complex (Brassicaceae). Plants have a long history of being edaphic specialists and

396 soils have unique micro-climates and biotic factors that plants must adapt to.

398 Constitutive defenses may be a better defense against herbivores in tropical plant systems-

399 We found little support for herbivory being solely responsible for up- or down-regulating

400 plant chemical defenses. Herbivore damage caused $P$. imperiale to reduce the amount of

401 defensive compounds except when plants are exposed to high levels of herbivory and when they

402 are also grown on residual soils with nutrient additions and high light (Figs. 4-5). It is important

403 to note that low light conditions and year-round pest pressure are the norm in deeply shaded

404 tropical forest understories (Dyer and Letourneau 1999). Thus, induced defenses caused by

405 herbivory may not apply to certain phytochemicals that are constitutive defenses and remain at

406 high concentrations even when herbivores are absent (Bixenmann et al. 2016) and depend more

407 on plant ontogenetic development (Fuchs and Bowers 2004, Quintero and Bowers 2011, Barton

408 2013). We found, however, that nutrients and soil type were more consistent predictors of

409 defensive state. Another experiment with three Piper species at La Selva found no effect of 
410 herbivory on chemical defenses (Fincher et al. 2008). This suggests that some Piper plants in

411 tropical forests produce and maintain a constant level of constitutive chemical defenses that are

412 not inducible based exclusively on herbivory. Our results are in contrast to plant communities

413 that experience seasonal pulses in pest pressure where plants rely more heavily on induced

414 defenses for protection against herbivores (Williams \& Whitham 1986, Karban et al. 1997,

415 Agrawal 2001). Chemical defenses expressed by the plant are not a unified trait that can be

416 defined simply as herbivore-induced or not, and our results show that limiting resources are a

417 major component to plant defense strategies.

\section{Conclusion}

419 Plants simultaneously experience multiple and sometimes contrasting pressures and growing 420 conditions. Growth and defensive phytochemistry of $P$. imperiale exhibited tradeoffs depending 421 on light availability and interactions among nutrient availability, contrasting soil types and

422 presence or absence of herbivores. Our results demonstrate that light and soil heterogeneity, 423 which vary from very small to larger spatial scales, can have dramatic effects on the 424 phytochemical profile of plants, leading to intraspecific variation in defense and palatability.

425 Whether or not this intraspecific variation found in $P$. imperiale leads to variation in plant fitness 426 has yet to be tested, but implications from our data show that there most likely is not one

427 phenotype that will be universally fit. Furthermore, plant defense theory and experimental 428 designs focused on intraspecific chemical variation should consider multiple drivers of plasticity 429 simultaneously rather than in isolation. These results are relevant to terrestrial and aquatic 430 ecosystems alike, and a renewed focus on issues such as elemental stoichiometry and allocation 431 to defense in terrestrial and aquatic systems (e.g., Sardans et al. 2012) will yield a clearer picture 432 of how and why anti-parasite defense varies intraspecifically across the landscape. 
433 Acknowledgments: We would like to thank Diego Salazar Amoretti and Bernal Matarrita

434 Carranza for help establishing Piper imperiale individuals in the shade-house at La Selva.

435 Furthermore, Earthwatch volunteers were a huge help with harvesting plants. The authors have

436 no conflict of interest to report.

\section{Declarations}

438 Funding: This research was funded by the Hitchcock Center for Chemical Ecology and 439 supported by the Garden Club of America Award in Tropical Botany and by the National

440 Science Foundation grant numbers DEB 1502059 and DEB 1442103.

441 Conflicts of interest: The authors have no conflicts of interest to declare.

442 Ethics approval: Not applicable.

443 Consent to participate: Not applicable.

444 Consent for publication: Not applicable.

445 Availability of data and material: This data has not been published elsewhere. Data is

446 available in Dryad repository (DOI \#) and on an open access website maintained by Lee A. Dyer

$447<\mathrm{http}: / /$ caterpillars.org/>.

448 Code availability: Not applicable.

449 References

450 Adler FR and Karban R (1994) Defended fortresses or moving targets? Another model of 451 inducible defenses inspired by military metaphors. The American Naturalist 144: 813$452 \quad 832$.

453 Agrawal AA and Karban R (1999) Why induced defenses may be favored over constitutive $454 \quad$ strategies in plants. The ecology and evolution of inducible defenses (pp. 45-61). Eds. R. 455 Tollrian and C.D. Harvell. Princeton University Press, Princeton, NJ. 
Agrawal AA (2001) Phenotypic plasticity in the interactions and evolution of species. Science 294: 321-326.

Barbosa et al. (2009) Associational resistance and associational susceptibility: having right or wrong neighbors. Annual Review of Ecology, Evolution, and Systematics 40: 1-20.

Bever JD (2003) Soil community feedback and the coexistence of competitors: conceptual frameworks and empirical tests. New Phytologist 157: 465-473

Bryant et al. (1983) Carbon/nutrient balance of boreal plants in relation to vertebrate herbivory. Oikos 40: 357-368

Cacho NI and Strauss SY (2014) Occupation of bare habitats, an evolutionary precursor to soil specialization in plants. Proceedings of the National Academy of Sciences 111: 1513215137.

Chazdon RL and Fetcher N (1984) Light environments of tropical forests. In Physiological ecology of plants of the wet tropics (pp. 27-36). Eds. Medina, Mooney, and VázquezYánes. Springer, Dordrecht.

Clark et al. (1998) Edaphic variation and the mesoscale distribution of tree species in a neotropical rain forest. Journal of Ecology 86: 101-112.

Clark DA (2013) NPP Tropical Forest: La Selva, Costa Rica, 1975-1994, R1. Data set. Available on-line [http://daac.ornl.gov] from Oak Ridge National Laboratory Distributed Active Archive Center, Oak Ridge, Tennessee, USA. doi:10.3334/ORNLDAAC/218.

Cohen J (1994) The earth is round. American Psychologist 49: 997.

Coley et al. (1985) Resource availability and plant antiherbivore defense. Science 230: 895-899.

Cronin G and Hay ME (1996) Induction of seaweed chemical defenses by amphipod grazing. 
Ecology 77: 2287-2301

480 Dicke M and Hilker M (2003) Induced plant defences: from molecular biology to evolutionary ecology. Basic and Applied Ecology 4: 3-14.

482 Dodson et al. (2000) Cenocladimide, a dihydropyridone alkaloid from Piper cenocladum. Phytochemistry 53: 51-54.

484 Dyer LA and Letourneau DK (1999) Relative strengths of top-down and bottom-up forces in a tropical forest community. Oecologia 119: 265-274.

Dyer et al. (2001) Trade-offs in antiherbivore defenses in Piper cenocladum: ant mutualists versus plant secondary metabolites. Journal of Chemical Ecology 27: 581-592.

Dyer LA and Palmer AD. eds. (2004) Piper: a model genus for studies of phytochemistry, ecology, and evolution. New York: Kluwer academic/Plenum publishers.

Dyer et al. (2018) Modern approaches to study plant-insect interactions in chemical ecology. Nature Reviews Chemistry 2: 50-64.

Endara et al. (2017) Coevolutionary arms race versus host defense chase in a tropical herbivoreplant system. Proceedings of the National Academy of Sciences 114: E7499-E7505.

494 Feeny P (1976) Plant apparency and chemical defense. In Biochemical interaction between plants and insects (pp. 1-40). Springer, Boston, MA.

496 Fincher et al. (2008) Inter-and intraspecific comparisons of antiherbivore defenses in three species of rainforest understory shrubs. Journal of Chemical Ecology 34: 558-574.

498 Fine et al. (2004) Herbivores promote habitat specialization by trees in Amazonian forests. Science 305: 663-665.

Fine etal. (2006) The growth-defense trade-off and habitat specialization by plants in Amazonian forests. Ecology 87: S150-S162. 
502 Fink P (2007) Ecological functions of volatile organic compounds in aquatic systems. Marine 503 and Freshwater Behaviour and Physiology 40: 155-168.

504 Fowler SV and Lawton JH (1985) Rapidly induced defenses and talking trees: the devil's advocate position. The American Naturalist 126: 181-195.

Gentry AH. ed. (1990) Four Neotropical Rainforests. Yale University Press, New Haven, Connecticut.

Glassmire et al. (2016) Intraspecific phytochemical variation shapes community and population structure for specialist caterpillars. New Phytologist 212: 208-219.

510 Glassmire et al. (2017) The soil mosaic hypothesis: a synthesis of multi-trophic diversification via soil heterogeneity. Ideas in Ecology \& Evolution 10: 20-26.

512 Glassmire et al. (2019) Proximity to canopy mediates changes in the defensive chemistry and herbivore loads of an understory tropical shrub, Piper kelleyi. Ecology Letters 22: 332-

515 Griffin et al. (2017) Pervasive interactions between foliar microbes and soil nutrients mediate leaf production and herbivore damage in a tropical forest. New Phytologist 216: 99-112.

517 Hahn PG and Maron JL (2016) A framework for predicting intraspecific variation in plant defense. Trends in Ecology \& Evolution 31: 646-656.

519 Hahn et al. (2018) Population variation, environmental gradients, and the evolutionary ecology of plant defense against herbivory. The American Naturalist 193: 20-34.

521 Hamilton et al. (2001) The carbon-nutrient balance hypothesis: its rise and fall. Ecology Letters 4: 86-95.

523 Hansen et al. (2017) Patterns in parasitism frequency explained by diet and 524 immunity. Ecography 40: 803-805. 
525 Hay ME (2002) The next wave in aquatic chemical ecology. Journal of Chemical Ecology 28:1-897.

527 Herms DA and Mattson WJ (1992) The dilemma of plants: to grow or defend. The Quarterly $528 \quad$ Review of Biology 67: 283-335.

529 Hunter MD and Price PW (1992) Playing chutes and ladders: heterogeneity and the relative roles of bottom-up and top-down forces in natural communities. Ecology 73: 724-732.

531 Hunter MD ed. (2016) The Phytochemical Landscape: Linking Trophic Interactions and Nutrient Dynamics. Princeton University Press.

533 Karban et al. (1997) The benefits of induced defenses against herbivores. Ecology 78: 13511355.

535 Kessler A and Kalske A (2018) Plant secondary metabolite diversity and species interactions. Annual Review of Ecology, Evolution, and Systematics 49: 115-138.

537 Koricheva J (2002) The carbon-nutrient balance hypothesis is dead; long live the carbon-nutrient balance hypothesis? Oikos 98: 537-539.

539 Laliberté E et al. (2013) How does pedogenesis drive plant diversity? Trends in Ecology and $540 \quad$ Evolution 28: 331-340.

541 Lerdau M and Coley PD (2002) Benefits of the carbon-nutrient balance hypothesis. Oikos 98: 534-536.

543 Loomis W (1932) Growth-differentiation balance vs. carbohydrate-nitrogen ratio. Proceedings of the American Society for Horticultural Science 29:240-245

545 Massad et al. (2012) Costs of defense and a test of the carbon-nutrient balance and growth546 differentiation balance hypotheses for two co-occurring classes of plant defense. PLoS $547 \quad$ One 7: e47554. 
548 Meiners et al. (2017) Soil microbial communities alter leaf chemistry and influence allelopathic potential among coexisting plant species. Oecologia 183: 1155-1165.

550 Misiewicz TM and Fine PVA (2014) Evidence for ecological divergence across a mosaic of soil types in an Amazonian tropical tree: Protium subserratum (Burseraceae). Molecular Ecology 23: 2543-2558.

553 Moore et al. (2014) Explaining intraspecific diversity in plant secondary metabolites in an ecological context. New Phytologist 201: 733-750.

555 Montgomery R and Chazdon RJ (2002) Light gradient partitioning by tropical tree seedlings in 556 the absence of canopy gaps. Oecologia 131: 165-174.

557 Morrison WE and Hay ME (2011) Induced chemical defenses in a freshwater macrophyte suppress herbivore fitness and the growth of associated microbes. Oecologia 165: 427-

560 Myers JH (1988) Can a general hypothesis explain population cycles of forest Lepidoptera? Advances in Ecological Research 18: 179-242.

562 Pasquini et al. (2015) Lianas always outperform tree seedlings regardless of soil nutrients: results 563 from a long-term fertilization experiment. Ecology 96: 1866-1876.

564 Pendergast et al. (2013) Belowground biotic complexity drives aboveground dynamics: a test of 565 the soil community feedback model. New Phytologist 197: 1300-1310.

566 Plummer M (2021) Rjags: Bayesian Graphical Models using MCMC. R package version 567 4-12. https://CRAN.R-project.org/package=rjags

568 R Core Team (2018) R: A language and environment for statistical computing. R Foundation for 569 Statistical Computing, Vienna, Austria.

570 Rhoades DF and Cates RG (1976) Toward a general theory of plant antiherbivore chemistry. 
In Biochemical interaction between plants and insects (pp. 168-213). Springer, Boston, MA.

Rhoades DF (1979) Evolution of plant chemical defense against herbivores. Herbivores: their interaction with secondary plant metabolites (pp. 3-54). Academic Press, Inc., New York, NY.

Richards et al. (2010) Synergistic effects of amides from two Piper species on generalist and specialist herbivores. Journal of Chemical Ecology 36: 1105-1113.

Richards et al. (2015) Phytochemical diversity drives plant-insect community diversity. Proceedings of the National Academy of Sciences 112: 10973-10978.

Rosseel Y (2012) Lavaan: An R package for structural equation modeling. Journal of Statistical Software 48: 1-36 < http://www.jstatsoft.org/v48/i02/>.

Saikkonen et al. (2013) Chemical ecology mediated by fungal endophytes in grasses. Journal of Chemical Ecology 39: 962-968.

Sancho F and Mata R (1987) Estudio detallado de suelos: Estacion Biologica 'La Selva.' Organization for Tropical Studies, San Jose, Costa Rica.

Sardans et al. (2012) The elemental stoichiometry of aquatic and terrestrial ecosystems and its relationships with organismic lifestyle and ecosystem structure and function: a review and perspectives. Biogeochemistry 111: 1-39.

Simms EL (1992) Costs of plant resistance to herbivory. Plant resistance to herbivores and pathogens: ecology, evolution, and genetics (pp. 392-425). University of Chicago Press, Chicago.

Smilanich et al. (2016) Does plant apparency matter? Thirty years of data provide limited 
support but reveal clear patterns of the effects of plant chemistry on herbivores. New Phytologist 210: 1044-1057.

595 Stamp N (2003) Out of the quagmire of plant defense hypotheses. The Quarterly Review of Biology 78: 23-55.

597 Stamp N (2004) Can the growth-differentiation balance hypothesis be tested rigorously? Oikos 107: 439-448.

599 Tabachnick BG and Fidell LS (1989) Profile analysis of repeated measures. Using multivariate statistics (pp. 437-505). Harper Collins, New York, NY.

601 Tabachnick et al. (2001) Using multivariate statistics (eds. Haper and Row, 5th edition). New 602 York, NY.

603 Tomlinson et al. (2016) Defense against vertebrate herbivores trades off into architectural and 604 low nutrient strategies amongst savanna Fabaceae species. Oikos 125: 126-136.

605 Torchiano M (2020) Package effsize: Efficient Effect Size Computation. doi:

606 10.5281/zenodo.1480624. R package version 0.8.0. <URL: https://CRAN.Rproject.org/package $=$ effsize $>$.

608 Turcotte MM and Levine JM (2016) Phenotypic plasticity and species

609 coexistence. Trends in Ecology \& Evolution 31: 803-813.

610 Underwood et al. (2014) A conceptual framework for associational effects: when do neighbors 611 matter and how would we know? The Quarterly Review of Biology 89: 1-19.

612 Van Alstyne KL (1988) Herbivore grazing increases polyphenolic defenses in the intertidal 613 brown alga Fucus distichus. Ecology 69: 655-663.

614 van der Putten et al. (2013) Plant-soil feedbacks: the past, the present, and future challenges. 615 Journal of Ecology 101: 265-276. 
616 Williams AG and Whitham TG (1986) Premature leaf abscission: an induced plant defense against gall aphids. Ecology 67: 1619-1627.

618 Wright et al. (2011) Potassium, phosphorus, or nitrogen limit root allocation, tree growth, or $619 \quad$ litter production in a lowland tropical forest. Ecology 92: 1616-1625. 


\section{Figure captions}

620 Figure 1. Testing the degree to which resource availability, contrasting soil type, and herbivory

621 generate intraspecific chemical variation via estimating tradeoffs between growth and defensive

622 compounds. The top panel depicts the chemical structures of the two nitrogen-based defensive

623 compounds that were quantified in this experiment. The imide compounds, (1) sintenpyridone

624 and (2) piplaroxide, are biosynthetically related, where (1) is a precursor to (2). The bottom panel

625 depicts the Piper imperiale individual plants used in this experiment with treatment level

626 combinations. We used a factorial design and exposed established plant fragments to random

627 treatment level combinations of either (a) low or high levels of light, (b) alluvial (light brown in

628 image) or residual soils (black in image), and (c) herbivory (Eois herbivore shown feeding on

629 leaf). (d) Above- and below-ground plant biomass were harvested and weighed for each plant.

630 Figure 2. Comparisons of above- and below- ground plant biomass along an herbivory gradient

631 from $0-30 \%$ are shown here. Low light is represented by the gray panels and high light is

632 represented by the yellow panels. The green line represents aboveground biomass and the purple

633 line represents belowground biomass. Herbivory generally decreased above-ground plant

634 biomass and residual soil facilitated reductions in above- and below-ground plant biomass.

635 Figure 3. Protein expressed as percentage dry weight in leaf tissue along an herbivory gradient

636 from $0-30 \%$. Low light is represented by the gray panels and high light is represented by the

637 yellow panels. In low light conditions, herbivory caused the greatest reductions of protein

638 content, when plants were established in residual soils and with high nutrient additions.

639 Conversely, in high light conditions, herbivory caused large increases in protein content,

640 especially when plants were established in old alluvial soils and with enhanced nutrient

641 additions. 
642 Figure 4. Path analysis examining the direct and indirect effects of herbivory, contrasting soil

643 type, and nutrient enrichments on plant growth and chemistry. The grey panel represents the

644 plants established under low light levels $\left(\chi^{2}=0.09 ; \mathrm{df}=1 ; P>0.77\right)$, while the yellow panel

645 represents the plants established under high light levels $\left(\chi^{2}=3.36 ; \mathrm{df}=1 ; P=0.07\right)$. Positive

646 relationships have arrow heads and negative relationships have blunt ends.

647 Figure 5. Defensive compounds expressed as percent dry weight in leaf tissue along an

648 herbivory gradient from $0-30 \%$. Low light is represented by the gray panels and high light is

649 represented by the yellow panels. The purple line represents piplaroxide and the green line

650 represents sintenpyridone. Sintenpyridone generally had stable levels no matter nutrient

651 additions, herbivory, and contrasting soil type. Piplaroxide, however, fluctuated more and

652 depended on the treatment combination. Herbivory caused piplaroxide to decrease in plants

653 established in residual soils and with low nutrient additions under both low and high light levels. 
Figures

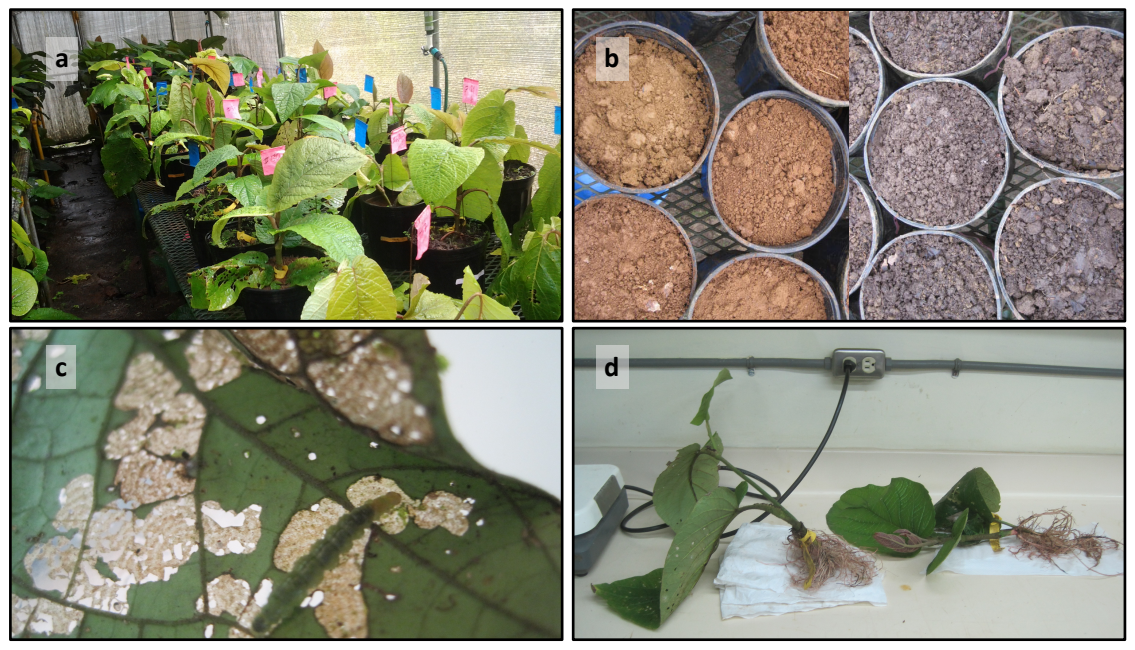

654

655 Figure 1. 


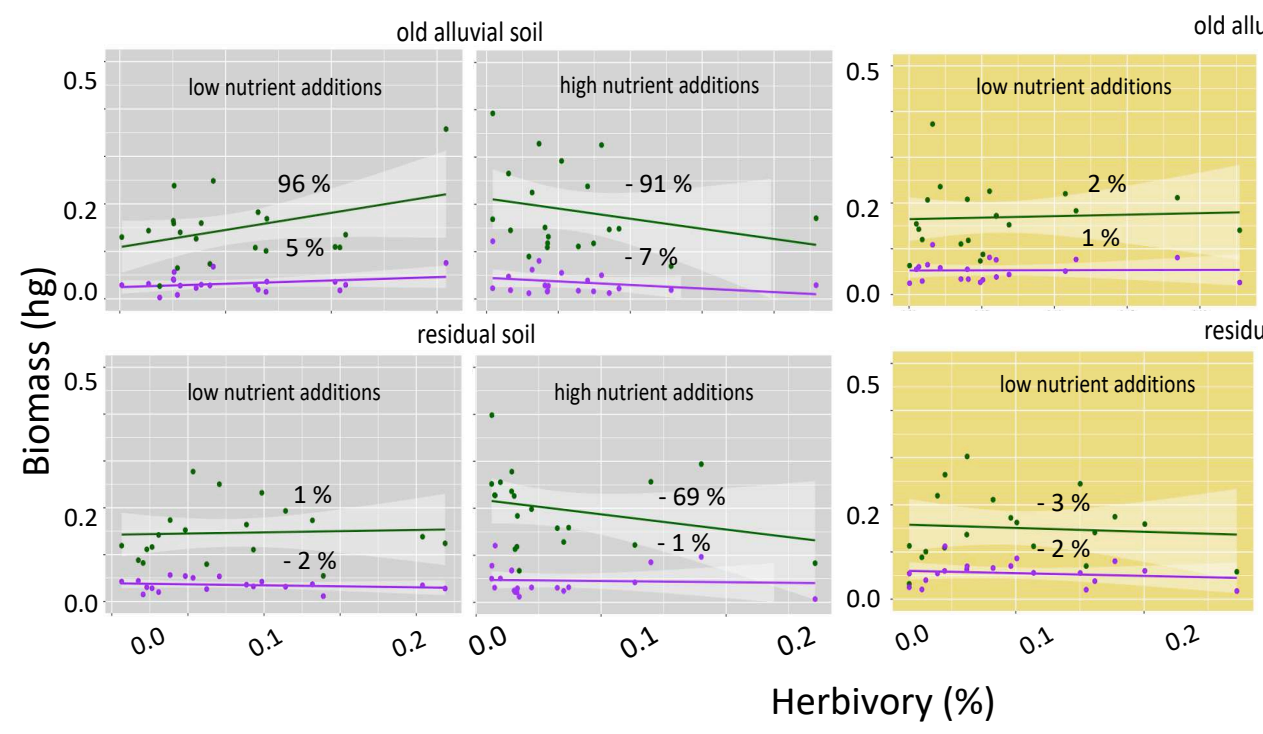

656

\section{Figure 2.}

658
Id alluvial soil

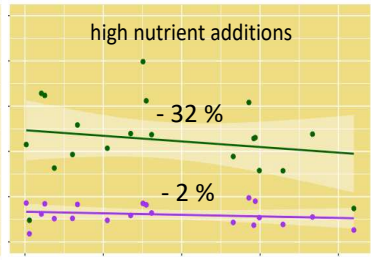

residual soil

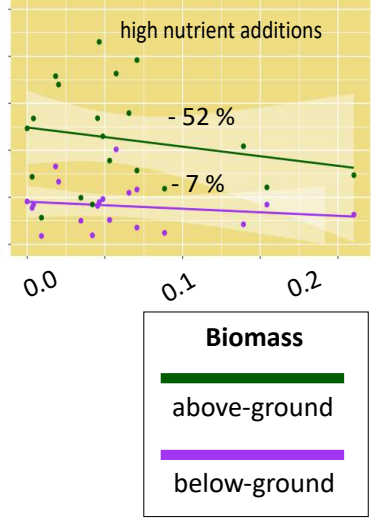




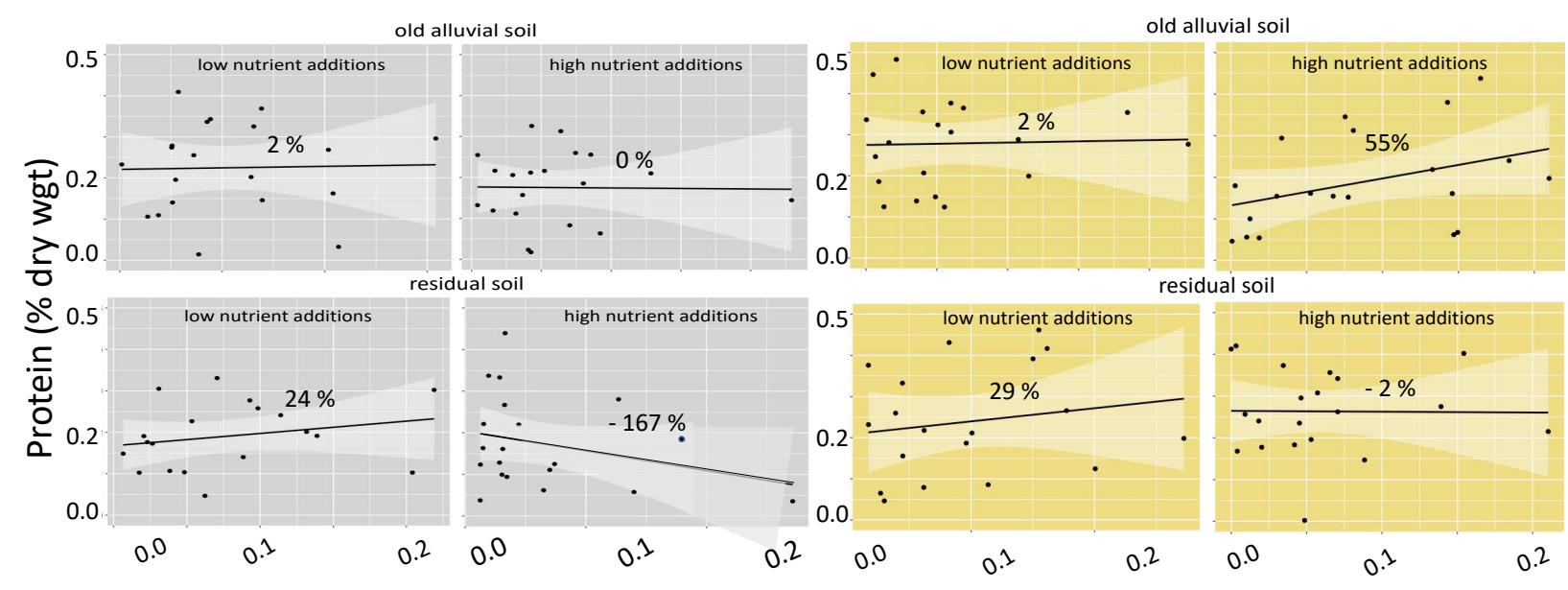

Herbivory (\%)

659

$660 \quad$ Figure 3. 


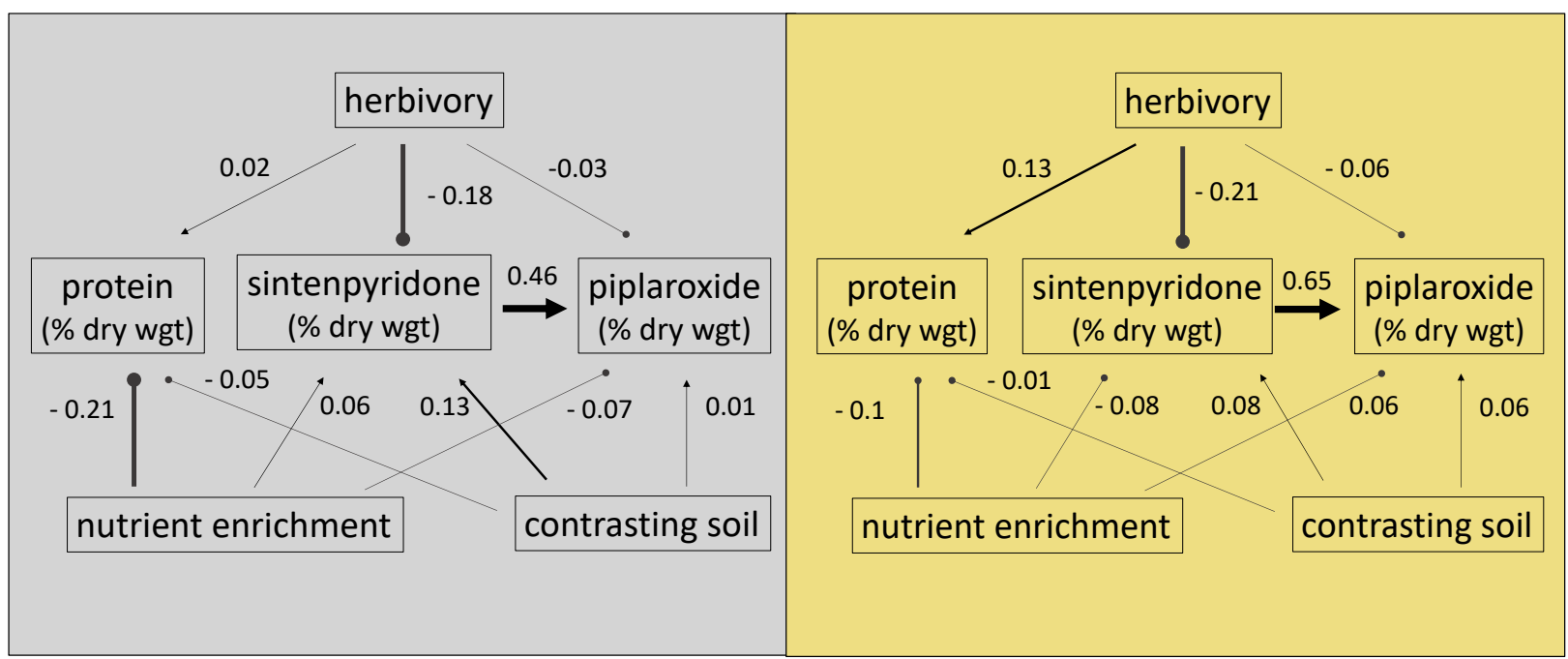

661

662 Figure 4. 


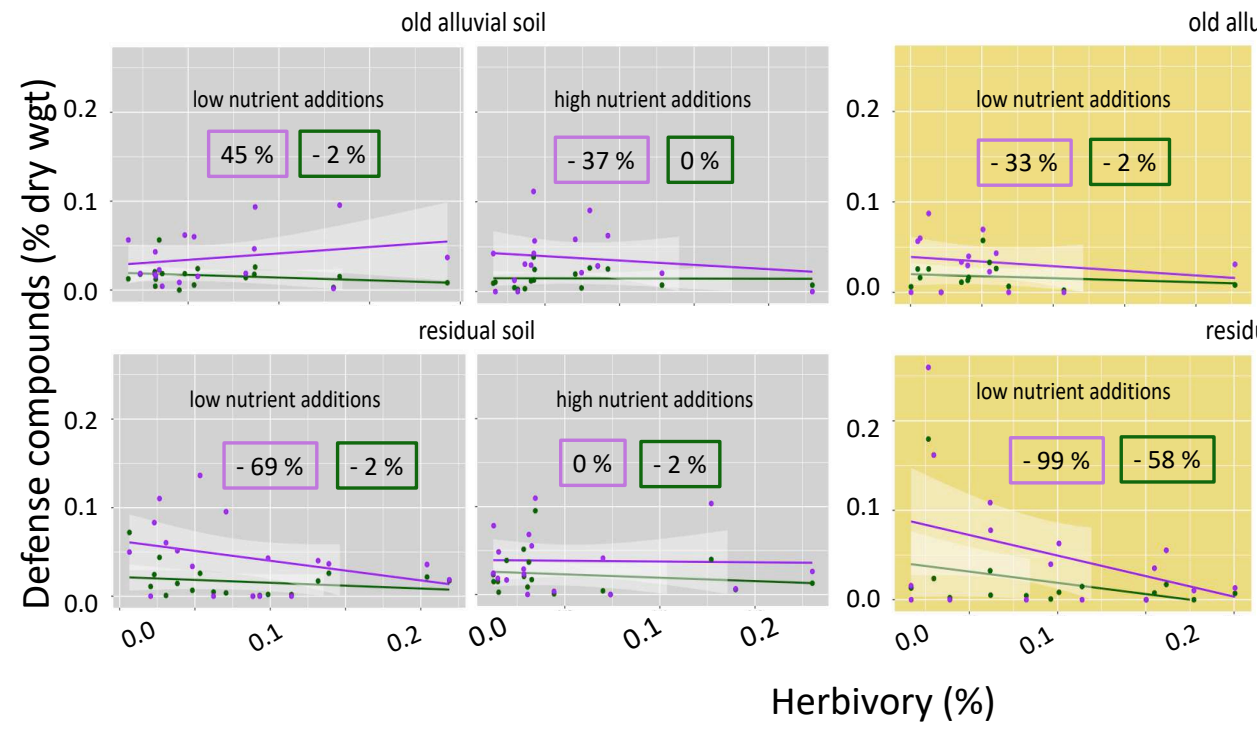

663

\section{Figure 5.}

old alluvial soil

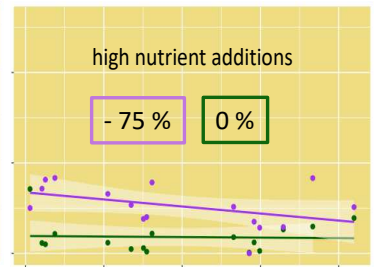

sidual soil

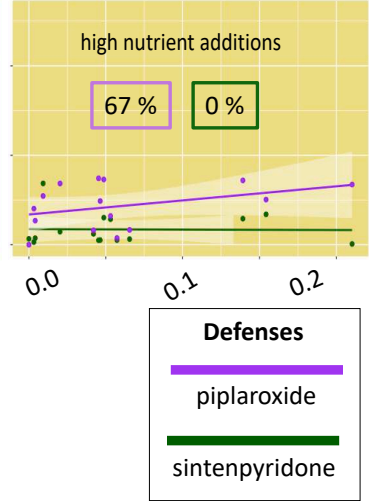




\section{Supplementary Files}

This is a list of supplementary files associated with this preprint. Click to download.

- GlassmireESM1.pdf 